\title{
IMPACTO INICIAL DEL ETIQUETADO DE ADVERTENCIA EN LA MODIFICACIÓN DE LOS HÁBITOS ALIMENTARIOS DE LA POBLACIÓN ADULTA EN MÉXICO
}

\section{INITIAL IMPACT OF WARNING LABELS ON FOOD HABITS OF ADULT CONSUMERS IN MEXICO}

\author{
Ana María Paredes-Arriaga ${ }^{1 *}$ (D); María Cristina-Alicia Velázquez-Palmer ${ }^{2}$ (D); Mónica Prats-Robles ${ }^{3}$ (D). \\ 1.Universidad Autónoma Metropolitana. anamarparedes2000@yahoo.com.mx \\ 2. Universidad Autónoma Metropolitana.mcvpalmer@yahoo.com.mx \\ 3. Universidad Autónoma Metropolitana.monica_prats@hotmail.com \\ *Autor de Correspondencia: Ana María Paredes-Arriaga correo electrónico: anamarparedes2000@yahoo.com.mx
}

\section{RESUMEN}

El sobrepeso y la obesidad constituyen un problema de salud pública en México, lo que ha impulsado al gobierno mexicano a desarrollar una serie de acciones tendientes a su resolución. Entre las más recientes se encuentran las modificaciones a la Norma 051 que regula el etiquetado de alimentos y bebidas pre-envasados. En este contexto, el presente estudio tuvo como objetivo conocer el impacto inicial del etiquetado frontal en los hábitos de consumo de alimentos y bebidas pre-envasados en la población adulta, desde la perspectiva de la mercadotecnia social. Con este fin, se realizó un estudio ex post facto de corte transversal. La información fue recopilada mediante un cuestionario aplicado en línea a una muestra de 514 consumidores de 18 años en adelante. Entre los hallazgos se destaca el que los consumidores identifican la presencia de sellos de advertencia en los productos, pero no se sienten motivados a cambiar sus hábitos y a dejar de comprar dichos productos. Por su naturaleza, la mercadotecnia social puede contribuir a la solución de esta problemática mediante campañas integrales, de las cuales ha adolecido el gobierno mexicano.

Palabras clave: NOM 051; sobrepeso y obesidad; mercadotecnia social.

Cómo citar:

Paredes-Arriaga, Ana María; Velázquez-Palmer, María Cristina-Alicia; Prats-Robles, Mónica. (2021). IMPACTO INICIAL DEL ETIQUETADO DE ADVERTENCIA EN LAMODIFICACIÓN DE LOS HÁBITOS ALIMENTARIOS DE LA POBLACIÓN ADULTA EN MÉXICO. Revista de Investigaciones Universidad del Quindio, 33(S1), 56-70. https://doi.org/10.33975/riuq. vol33nS1.483 


\begin{abstract}
Overweight and obesity have become a public health issue in Mexico. Among actions taken by the authorities to solve it, the Norm 051 that regulates labeling of packaged food and beverages has been modified recently. This study aims to know the initial impact of warning labels on adult consumers habits, from a social marketing perspective. A quantitative design was developed and 514 consumers of 18 years on were interviewed. Major findings show that consumers are aware of warning seals on the products, but are not motivated to change their habits, nor to stop buying them. Social marketing is a discipline that can contribute to design integral campaigns to solve this problem, since the actions taken by the Mexican authorities lack of an integral approach.
\end{abstract}

Keywords: NOM 051; overweight and obesity; social marketing.

\title{
INTRODUCCIÓN
}

La obesidad y el sobrepeso a nivel mundial se han convertido en un problema de salud pública que ha hecho necesaria la intervención de los gobiernos en sus diferentes niveles, para contrarrestar y detener los efectos negativos de estos padecimientos en la salud y calidad de vida de la población, así como por su efecto a nivel económico, derivado de los costos de atención y de la baja en la productividad de las personas afectadas.

En el caso de México, desde la edición de 2006 de la Encuesta Nacional de Salud y Nutrición (ENSANUT), que se lleva a cabo periódicamente, se observa una tendencia creciente en la incidencia de obesidad y sobrepeso de la población en general, tanto infantil como adulta. Esto ha propiciado que desde el sexenio del presidente Felipe Calderón, se hayan implementado diferentes acciones tendientes a modificar los hábitos alimentarios de los mexicanos. Entre estas estrategias destacan los cambios en el etiquetado de los alimentos y bebidas procesados, a fin de que el consumidor pueda tomar decisiones informadas con relación a los alimentos que integran su dieta diaria.

De esta manera, en un primer momento se hizo obligatoria la inclusión del contenido nutrimental en la etiqueta/empaque de los productos y posteriormente, se incluyeron leyendas relacionadas con el contenido de grasas, azúcares, sodio y calorías. Sin embargo, diversos grupos que han trabajado a favor de lograr una mejor nutrición entre la población, como Alianza por la Salud Alimentaria, consideraron que estas etiquetas eran poco claras y no cumplían su cometido de alertar a los consumidores sobre el valor nutrimental de los alimentos. Así, en noviembre de 2019 se aprueba el etiquetado frontal de advertencia, conformado por sellos en los que se alerta por el contenido excesivo de azúcares, sodio, grasas, calorías. Estos cambios forman parte de la NOM-051 en la que además se incluyen modificaciones relacionadas con el cálculo y evaluación de los nutrimentos críticos.

En este contexto, la presente investigación tuvo como objetivo conocer el impacto inicial del etiquetado frontal en los hábitos de consumo de alimentos y bebidas procesadas en la población adulta, desde la perspectiva de la mercadotecnia social. 
Problemática del sobrepeso, la obesidad, y la diabetes en México.

José Ángel Gurría. Secretario General de la Organización para la Cooperación y el Desarrollo Económicos (OCDE), durante el Seminario Sobrepeso, Obesidad y Diabetes en enero de 2020, advirtió que "la obesidad en México es un problema muy severo, un tema ético y económico, que está afectando la esperanza de vida de los mexicanos" (Poder del consumidor, 2020: s/p).

En este sentido, en el programa Simbiosis de TV UNAM, la Dra. Shamah, directora adjunta del Centro de Investigación en Evaluación y Encuestas (CIEE) del Instituto Nacional de Salud Pública (INSP); comentó que en México el sobrepeso y la obesidad siguen representando un problema creciente, que no se detiene sin importar la zona, ya sea rica, pobre, rural o urbana (INSP, 2020). Lo anterior queda de manifiesto en la Encuesta Nacional de Salud y Nutrición (ENSANUT) 2018 (Gráfica1), donde se aprecia que de 2012 a 2018, tanto en mujeres como en hombres, hubo incremento en sobrepeso y en obesidad (ENSANUT, 2018).

Gráfica 1. Sobrepeso y obesidad en población de 20 y más años Sobrepeso y obesidad en población de 20 y más años

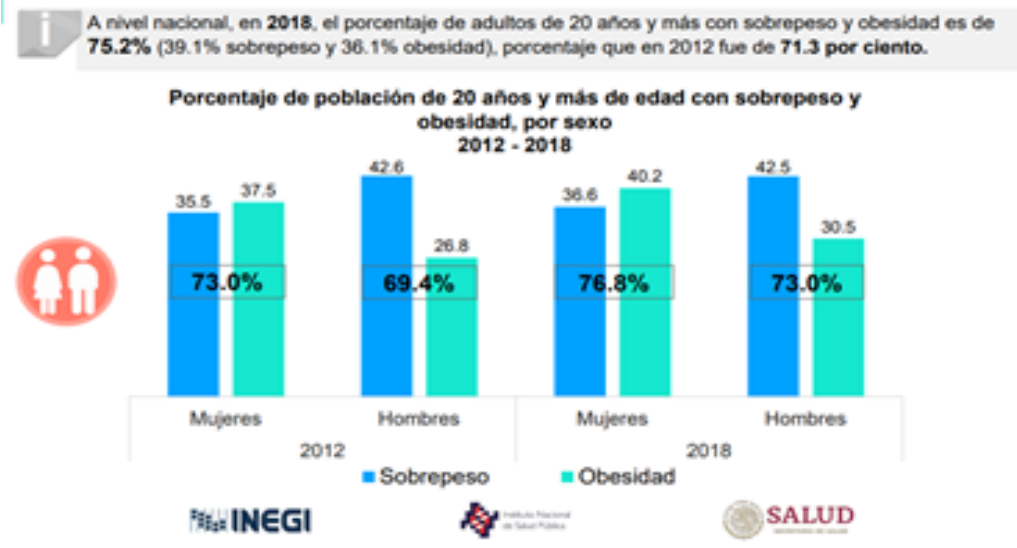

Fuente: ENSANUT 2018

Las altas tasas de obesidad y sobrepeso traen como consecuencia enfermedades como la hipertensión y la diabetes; esta última causa una tasa de hospitalización de 248.5 por cada 100 mil habitantes, que representa más del doble que el promedio en Latinoamérica que es de 122.5, y de 128.9 en la región de la OCDE (Villanueva, 2020).

En el 2016, en México fallecieron 105,572 personas por problemas relacionados a la diabetes, lo que la coloca como la segunda causa de muerte a nivel nacional, de acuerdo con información del INEGI. El costo de la diabetes para nuestro país suma cientos de millones de dólares al año. En 2016, el IMSS gastó \$315 millones de dólares, el ISSSTE \$89 y la Secretaría de Salud llegó a \$209 dando un total de \$614 millones de dólares (Alianza por la salud alimentaria, 2019).

Simón Barquera, director del área de investigación en Políticas y Programas de Nutrición del Instituto Nacional de Salud Pública (INSP), señala en un artículo escrito en coautoría con Mariel White, que "el presupuesto destinado a la prevención de la obesidad no solo se ha estancado, sino que los fondos para este rubro, se reducen año con año, lo que refleja la falta de voluntad de México para lidiar de manera efectiva con la obesidad y con las comorbilidades que la acompañan" (Obesity, 2018:1530). Asimismo, hace énfasis en que es necesario hacer cambios profundos e inmediatos en la estrategia del gobierno 
mexicano para resolver el problema, el cual ha sido declarado como emergencia epidemiológica.

En la ENSANUT 2018 se reporta que en 2012 había 6.4 millones de mexicanos con diagnóstico médico previo de diabetes y en sólo 6 años se incrementó a 8.6 millones (Gráfica 2).

Gráfica 2. Diabetes en población de 20 años y más años

\section{Diabetes en población de 20 años y más años}

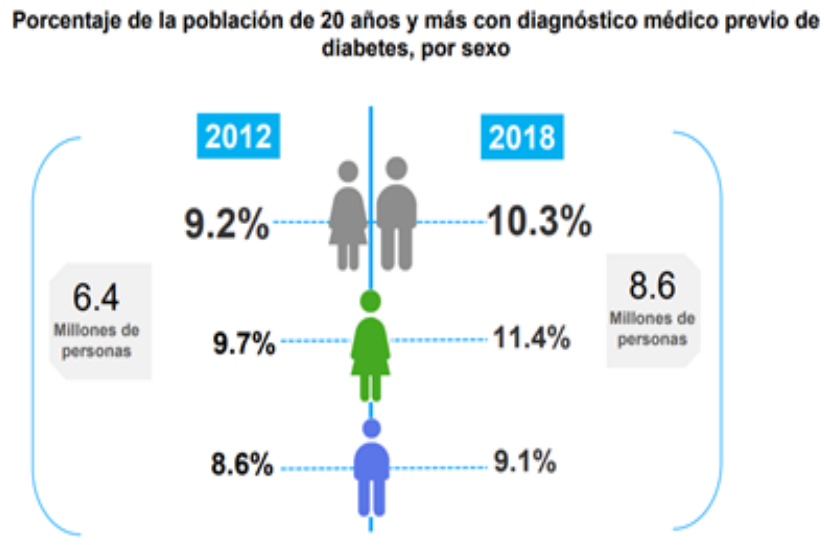

Fuente: ENSANUT 2018

Una de las causas de la obesidad y sobrepeso es principalmente el desequilibrio entre la ingesta de productos de alto contenido calórico y la vida sedentaria, lo que ha provocado un incremento en enfermedades como la diabetes, es decir, que dichos padecimientos tienen una relación directa con la alimentación, más en específico con el alto consumo de azúcares añadidos, grasas saturadas y sal, que se encuentran en los productos ultraprocesados y en las bebidas azucaradas. En este sentido, en la ENSANUT 2018, se señala que los productos no recomendables que más se consumen en nuestro país son: las bebidas no lácteas endulzadas, seguidas de las botanas, dulces y postres (Gráfica 3).

Gráfica 3. Consumo de alimentos No recomendables

\section{Consumo de alimentos No Recomendables}

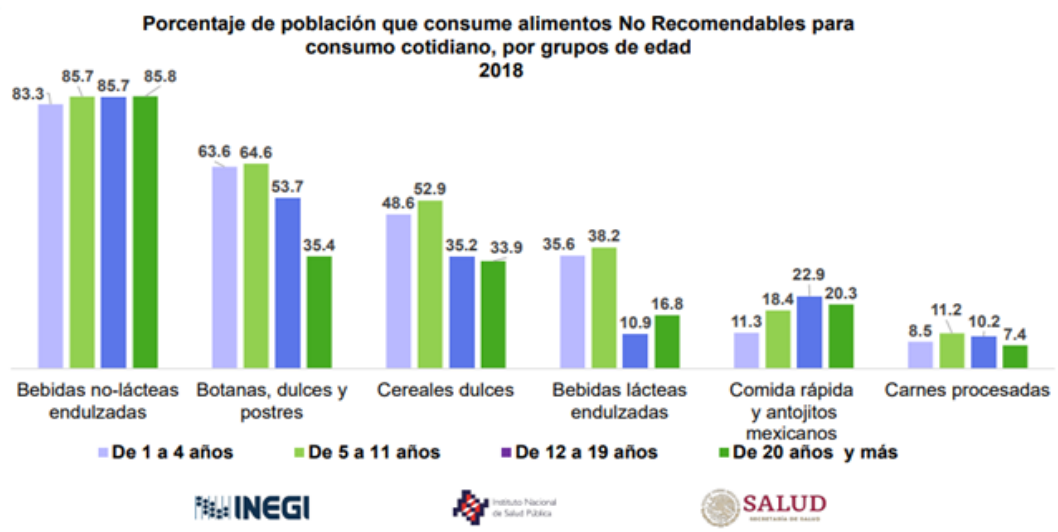

Fuente: ENSANUT 2018

Por lo anterior es necesario regular lo que propicia la mala alimentación y plantear acciones para solucionar de forma integral el problema de la diabetes; de acuerdo con algunas organizaciones como la Alianza por la salud alimentaria (2019), una manera de prevenirla es conociendo los nutrimentos críticos que contienen los productos procesados, a través de una etiqueta que de forma clara advierta 
a los consumidores del exceso de azúcares, grasas saturadas, sodio y calorías; y garantice el derecho a la información.

Por otro lado, las empresas globales, fabricantes de la comida "chatarra" y bebidas azucaradas, históricamente se han opuesto a las políticas dirigidas a combatir la mala alimentación, como lo señala la Alianza por la salud alimentaria (2019), que ha documentado este tipo de prácticas, entre las que se encuentran:

- Las recomendaciones emitidas por la Secretaría de Salud (SSa) en 2018 sobre bebidas para la población mexicana, que a través de la campaña "Jarra del Buen Beber" complementaba al "Plato del Buen Comer"; se obstaculizaron debido a la gran presión de la industria alimenticia, que bloqueó la difusión masiva, y decenas de miles de materiales impresos se quedaron sin distribuir.

- En 2010, el Consejo Coordinador Empresarial (CCE) junto con las asociaciones empresariales, estuvieron en contra del Acuerdo sobre los lineamientos de alimentos y bebidas en escuelas, y lograron que por años se mantuviera la minichatarra en las escuelas y que no se aplicaran cabalmente los lineamientos.

- En 2014, ConMéxico logró la aplicación obligatoria del etiquetado frontal Guías Diarias de Alimentación (GDA) que la propia industria había introducido en 2010-2011, en contubernio con la Comisión Federal de Protección contra Riesgos Sanitarios (Cofepris) y la Secretaría de Salud (SS), manteniendo en el engaño y la ignorancia a los consumidores mexicanos. ConMéxico, defiende el sistema de Etiquetado GDA con el argumento de que permite comparar entre productos, no obstante, al ser evaluado por investigadores libres de conflictos de interés, los resultados mostraron que la población no lo comprendía, lo consideraban complejo y no permitía la toma de decisiones informadas.

Ante este panorama surgió la inciativa, por parte de diversas organizaciones y expertos en el tema, de la necesidad de tener un etiquetado que proporcionara al consumidor información que le permitiera tomar decisiones mejor informadas respecto a su alimentación. Lo que finalmente condujo a la modificación de la Norma oficial 051, que entre otros aspectos contempla el uso del etiquetado frontal de advertencia en los productos envasados y bebidas no alcohólicas.

\section{Modificaciones a la Norma 051}

La Norma 051-SCFI/SSA1-2010 "Especificaciones generales de etiquetado de alimentos y bebidas no alcohólicas pre-envasados- Información comercial y sanitaria”, tiene como objetivo, proporcionar información comercial y sanitaria concreta y comprensible, que debe colocarse en la etiqueta frontal de los alimentos y bebidas de fabricación nacional o extranjera, para que el consumidor final esté en posibilidad de realizar una elección orientada que favorezca su bienestar presente y futuro.

El apartado que experimentó más reformas fue el 4 (Especificaciones), particularmente en los numerales concernientes al etiquetado anterior conocido como Guías Diarias de Alimentación (GDA). Cabe señalar, que una de las modalidades de productos en la que la Norma no es aplicable es aquella que se refiere a los alimentos y bebidas no alcohólicas vendidos a granel. Asimismo, algunas de las nuevas especificaciones que acompañan al nuevo etiquetado detallan que:

- Todo producto pre envasado que posea uno o más sellos de advertencia y/o leyenda de edulcorantes, no deberá contar en su etiqueta con "personajes infantiles, animaciones, dibujos 
animados, celebridades, deportistas o mascotas, elementos interactivos o descargas digitales, que estando dirigidos a niños inciten, promuevan o fomenten su consumo, compra o la elección" (DOF, 2020:14).

- La presentación de la declaración nutrimental debe considerar los valores de referencia acordes a la población mexicana y presentarse de acuerdo a la normatividad vigente.

- La declaración de la cantidad de proteínas, hidratos de carbono, grasas y sodio presentes en los alimentos y bebidas no alcohólicas pre-envasados se expresarán basándose en la medida de 100 $\mathrm{ml} \mathrm{o} 100 \mathrm{mg}$.

- El sistema de etiquetado frontal establece que la información nutrimental complementaria se registrará haciendo uso de los sellos que se muestran en la Imagen 1:
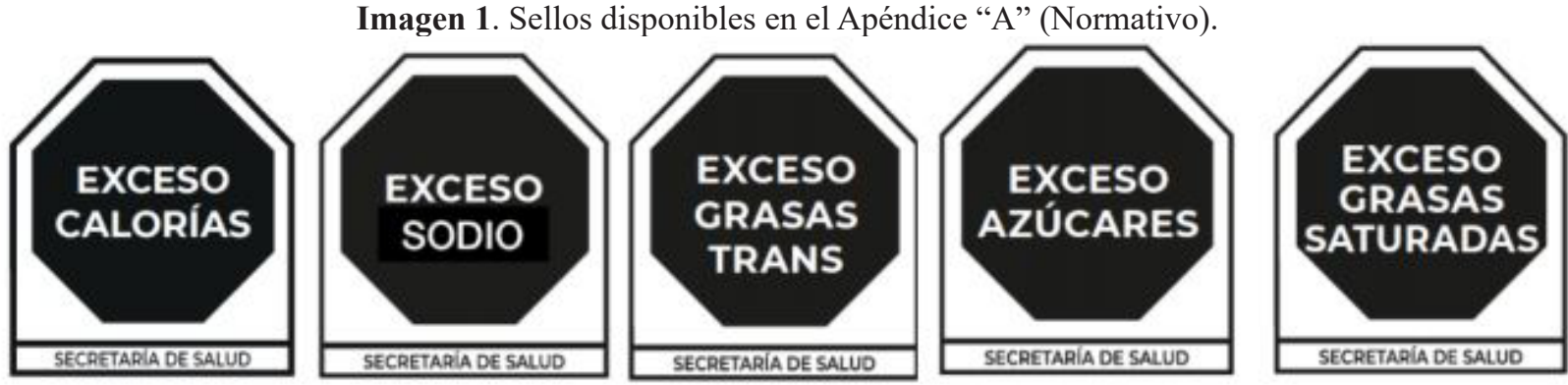

Fuente: Tomado de DOF, 2014:21

- Se establece que la sucesión de los sellos que deberán colocarse en la etiqueta en orden de izquierda a derecha son: "Exceso de calorías", "Exceso de azúcares", "Exceso de grasas saturadas”, "Exceso de grasas trans", "Exceso de sodio" los cuales se observan en la Imagen 2.

Imagen 2. Los cinco sellos de advertencia y su sucesión de acuerdo con el Apéndice "A" (Normativo)

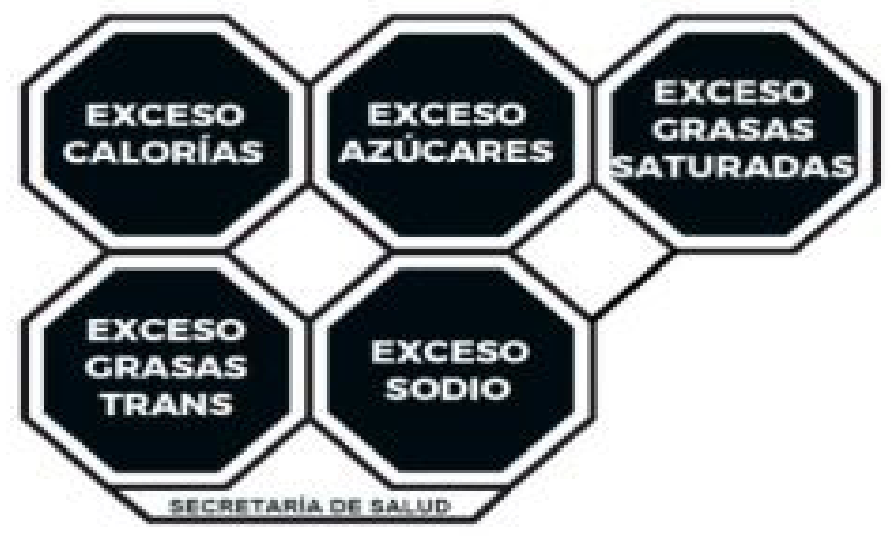

Fuente: Tomado de DOF, 2014:30

- Cuando sea necesario también podrá hacerse uso de las leyendas "Contiene cafeína evitar en niños", o bien "Contiene edulcorantes-no recomendable en niños".

- En caso de que el producto posea en su etiquetado alguno de los sellos o de las leyendas, no se deberán efectuar declaraciones de propiedades saludables.

- La verificación y vigilancia de la Norma están a cargo de la Procuraduría Federal del Consumidor, la Comisión Federal para la Protección contra Riesgos Sanitarios, y aquellas consideradas en la Ley Federal de Protección al Consumidor, la Ley General de Salud y la Ley General sobre Metrología y Normalización. 
La puesta en práctica de las modificaciones a la NOM-051 se llevará a cabo en tres fases. La primera inició el $1^{\circ}$ de octubre del 2020 , la segunda comenzará el $1^{\circ}$ de octubre de 2023 , y la tercera el $1^{\circ}$ de octubre de 2025 .

\section{El cambio a nivel de la sociedad y la mercadotecnia social}

Kotler (en Dibb y Carrigan, 2013) señala que en la sociedad existen individuos, grupos y organizaciones que tratan de influir para que ésta se oriente en una dirección determinada respecto a ciertas problemáticas sociales; y puede decirse que dichos grupos están comprometidos con la acción social y tratando de utilizar sus conocimientos, habilidades y poder para mejorar las condiciones de vida, así como la vida en el planeta en general. Asimismo, menciona que hay diferentes niveles para llevar a cabo la acción social los cuáles se muestran en la Figura 1 y se describen a continuación:

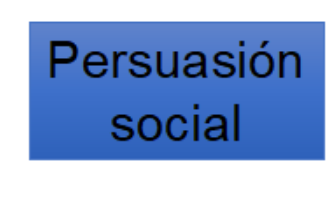

Figura 1. Niveles de la acción social Figura 1. Niveles de la accion social
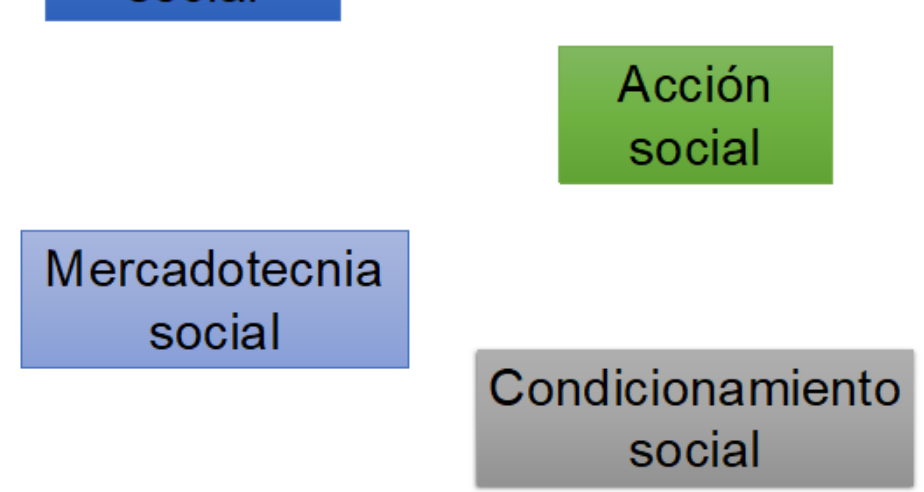

Fuente: elaboración propia con base en Dibb, S. y Carrigan, M. (2013). Social Marketing Transformed: Kotler Polonsky and Hastings reflect on social marketing in a period of social change. European Journal of Marketing. 47(9),
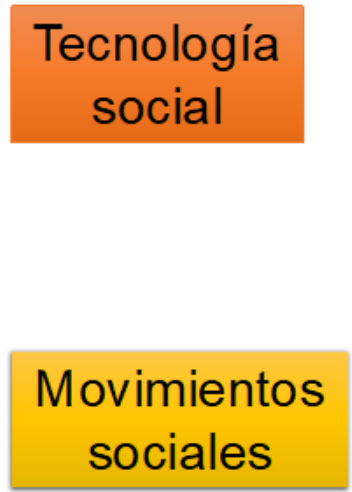

Persuasión social se refiere a las actividades dirigidas a influir en las actitudes y creencias y es la forma dominante de acción social. Puede realizarse a través de encuentros cara a cara o de medios en/fuera de línea. Si bien podría decirse que su objetivo final es el cambio de comportamiento, su meta inmediata es influir en actitudes y creencias como ya se ha mencionado.

- Tecnología social, está dirigida a influir en forma pasiva para lograr el cambio de comportamiento. A través de la tecnología se han desarrollado dispositivos como el cinturón de seguridad, que en algunos casos no le dan opción al conductor, ya que si no lo usa no avanza el auto.

- Mercadotecnia social, ésta tiene como objetivo, a diferencia de la anterior, influir de manera activa para cambiar el comportamiento de una audiencia objetivo. Se trata de una disciplina más formal que dispone de procesos y herramientas específicos para alcanzar el cambio en la conducta.

- Movimientos sociales, hacen referencia a acciones llevadas a cabo por grupos de presión para hacer frente a problemas sociales difíciles, que implican cambios de comportamiento en gran escala. Tal es el caso del cambio climático, la legalización de las drogas, los movimientos del consumidor, entre otros.

- Condicionamiento social, el cual promueve acciones para que las generaciones futuras actúen de manera "correcta". Un ejemplo es el del gobierno sueco que implementó toda una serie de actividades en su sistema de instituciones educativas para inculcar en los estudiantes el no fumar, 
no consumir drogas, reducir el consumo de alcohol, alimentarse en forma nutritiva y hacer ejercicio. La clave en los resultados obtenidos, que fueron positivos, radicó en la utilización de la teoría del aprendizaje social, que se basa en reforzar los comportamientos correctos con recompensas y los no adecuados con castigos (Kotler en Dibb y Carrigan, 2013).

De lo anterior se desprende que la mercadotecnia social está relacionada con el amplio campo de estudio del cambio social: sus manifestaciones, causas e influencias.

A través del tiempo, esta disciplina ha ido evolucionando de un enfoque orientado a influir en la aceptación de ideas sociales y en modificar actitudes, hasta centrarse en el cambio de comportamiento, ya que se trata no solo de generar una actitud positiva hacia una buena nutrición, sino realmente de modificar los hábitos alimentarios, por ejemplo. De esta manera, el objetivo de la mercadotecnia social es lograr un efecto en el comportamiento, ya sea eliminando o debilitando uno no deseado, o bien, fortaleciendo uno deseable; y alcanzando un beneficio para la sociedad en su conjunto, en lugar de una ganancia económica para una persona u organización. El éxito de una campaña de mercadotecnia social se mide en el corto plazo en función del número de conductas deseadas en las que se tuvo un efecto; y en el largo plazo, por el número de dichos comportamientos que se mantuvieron por un largo tiempo (Kotler en Dibb y Carrigan, 2013). Asimismo, hay que mencionar que una buena estrategia puede requerir de hasta diez años, ya que algunas prácticas de esta disciplina requieren de largos periodos para lograr el aprendizaje de los comportamientos deseados (Lusch y Vargo, 2006).

Para que un programa tendiente a lograr un cambio de conducta a nivel de la sociedad pueda ser calificado como mercadotecnia social debe considerar lo siguiente (Andreasen, 2002):

- El cambio de comportamiento es el punto central para diseñar y evaluar las intervenciones.

- Un uso continuo de la investigación de mercados para comprender a las audiencias antes y durante la intervención, así como para monitorear los resultados conforme se desarrolla el programa.

- Una cuidadosa segmentación del mercado para maximizar la eficiencia y eficacia en el uso de los recursos, que por lo general son escasos.

- La creación de intercambios atractivos para las audiencias. El comportamiento o los beneficios deben ser percibidos de manera inmediata y ser mayores a las conductas alternativas.

- Se diseñan estrategias que incluyen las cuatro P's de la mezcla tradicional de mercadotecnia. Así, se generan paquetes atractivos de beneficios (producto), al mismo tiempo que se busca minimizar los costos en la medida de lo posible (precio), facilitar el intercambio (plaza) y comunicar mensajes de alto impacto a través de los medios utilizados por las audiencias (promoción).

Asimismo, Andreasen (2005) señala que deben diseñarse estrategias en tres niveles:

- Hacia abajo, dirigidas a la audiencia objetivo, esto es los adultos con sobrepeso, los adolescentes con bulimia.

- Horizontal, que incluyen a los pares de la audiencia, esto es, los amigos, familiares, grupos de referencia que puedan ejercer una influencia positiva para lograr el comportamiento deseado.

- Hacia arriba, que comprende las organizaciones e instituciones que juegan un papel importante en mantener el comportamiento no deseado, como los fabricantes de alimentos con alto valor calórico; así como las que promueven la conducta deseada, en el caso de la obesidad infantil en México, el Instituto Mexicano del Seguro Social (IMSS), y las ONG como la Alianza por la Salud. 
La mercadotecnia social aborda problemáticas que además de demandar un cambio a nivel social para su solución, se caracterizan por involucrar a una diversidad de redes de actores, cuyas necesidades deben ser satisfechas, por lo que abarca una variedad de relaciones que se extienden a un contexto social más amplio que el de la mercadotecnia tradicional. Estas relaciones se dan simultáneamente a nivel micro (individual), grupal (grupos u organizaciones) y macro (sociedad) y se trata de lograr una sinergia entre los múltiples agentes de cambio: audiencia objetivo, comunidades, autoridades, diseñadores de políticas públicas, $\mathrm{ONG}$, iniciativa privada, entre otros, para lograr el comportamiento deseable (Domegan, 2008).

De esta manera, la mercadotecnia social ha sido utilizada en diferentes países como Estados Unidos de América, Reino Unido, Canadá, entre otros para una diversidad de problemáticas relacionadas con temas de salud, prevención de accidentes y adicciones, planificación familiar, así como el sobrepeso y la obesidad en niños y adultos (Stead, Hastings y McDermott, 2007).

\section{METODOLOGÍA}

Ante la tendencia del sobrepeso, la obesidad y la diabetes, las autoridades mexicanas han llevado a cabo diferentes acciones tendientes a lograr cambios en los hábitos alimentarios de la población, entre las que se encuentra la modificación de la NOM-051, en cuanto al etiquetado de alimentos y bebidas envasados. En este contexto, se desarrolló el presente estudio que tuvo como objetivo:

- Conocer el impacto inicial del etiquetado de advertencia en los hábitos de consumo de alimentos y bebidas procesadas en la población adulta, desde la perspectiva de la mercadotecnia social.

Del objetivo se derivó la siguiente hipótesis:

$\mathrm{Ho}_{1}$ : No hay relación entre el conocimiento del etiquetado de advertencia y la modificación de los hábitos de consumo de alimentos en los que aparecen los sellos.

\section{Diseño de investigación}

Para realizar la presente investigación se diseñó un estudio ex post facto, transversal, ya que se llevó a cabo en un punto en el tiempo. La información se recabó mediante un cuestionario directo estructurado, que se aplicó en línea, y que incluyó las siguientes secciones:

Cuadro 1. Secciones del cuestionario

\begin{tabular}{|c|c|}
\hline Sección & Número de preguntas \\
\hline Cambios en la información que aparece en las etiquetas & Cuatro \\
\hline $\begin{array}{c}\text { Impacto de los cambios en las etiquetas en cuanto a los } \\
\text { hábitos alimenticios }\end{array}$ & $\begin{array}{c}\text { Doce } \\
\text { Escala de Likert de cuatro categorías }\end{array}$ \\
\hline Perfil sociodemográfico de los entrevistados & Cinco \\
\hline
\end{tabular}

Fuente: elaboración propia

El universo bajo estudio estuvo conformado por hombres y mujeres de 18 años en adelante que viven en la Zona Metropolitana del Valle de México. Debido a la contingencia sanitaria, se utilizó un muestreo por conveniencia y como ya se mencionó, el cuestionario se aplicó en línea a 514 consumidores. 


\section{HALLAZGOS}

La presentación de los hallazgos se divide en tres apartados, en el primero se presentan los resultados en cuanto al nivel de conocimiento de los cambios en las etiquetas; en el segundo, los relacionados con el impacto de dichas modificaciones en el comportamiento de consumo; y en el tercero la prueba de la hipótesis.

\section{Conocimiento de los cambios en las etiquetas}

El 87.5\% de los entrevistados señaló haber notado un cambio en las etiquetas/envases de los alimentos y bebidas que consume, y de éste el 96.2\% identificó que el cambio consistía en la inclusión de unos sellos negros; por lo que puede afirmarse que los sellos de advertencia están alcanzando el objetivo de llamar la atención del consumidor. Ahora bien, al preguntar a los respondientes acerca de la información que éstos contienen, como se observa en el Cuadro 2 la mayoría identificó el contenido de los sellos que aparecen con mayor frecuencia en las etiquetas/envases de los productos, esto es, Exceso azúcares (93.4\%), Exceso sodio (91.1\%) y Exceso calorías (84.2\%).

A los consumidores que en principio no habían identificado la presencia de un cambio en las etiquetas/ envases, se les explicó en que consistía éste y se les preguntó sobre la información que aparecía en los sellos y leyendas de advertencia, y como se aprecia en el Cuadro 2, los sellos que se identificaron con mayor frecuencia son: Exceso azúcares (82.3\%), Exceso sodio (69.6\%) y Exceso calorías (67.1\%).

Los sellos de advertencia que identificaron los consumidores en ambos casos, son indicativos del tipo de alimentos que consumen, y que dependiendo del número de sellos que aparecen en el empaque pueden no ser tan saludables.

Cuadro 2. Información en los sellos y leyendas de advertencia

\begin{tabular}{|l|c|c|}
\hline \multicolumn{1}{|c|}{ Leyenda } & $\begin{array}{c}\text { Cambio identificado } \\
\text { espontáneamente } \\
\text { \% }\end{array}$ & $\begin{array}{c}\text { Cambio identificado } \\
\text { con ayuda } \\
\text { \% }\end{array}$ \\
\hline Exceso azúcares & 93.4 & 82.3 \\
\hline Exceso sodio & 91.1 & 69.6 \\
\hline Exceso calorías & 84.2 & 67.1 \\
\hline Exceso grasas saturadas & 56.7 & 41.8 \\
\hline Exceso grasas trans & 37.0 & 38.0 \\
\hline $\begin{array}{l}\text { Contiene edulcorantes. No recomendable en } \\
\text { niños }\end{array}$ & 17.1 & 10.1 \\
\hline Contiene cafeína. Evitar en niños & 15.9 & 15.2 \\
\hline
\end{tabular}

Fuente: elaboración propia

II. Impacto de las modificaciones en el comportamiento de consumo

Para conocer el impacto, se incluyeron tres grupos de variables: relacionadas con la lectura y contenido de las etiquetas, influencia de los sellos en la compra de los productos, y como medida para mejorar la alimentación de la gente. 


\section{Lectura y contenido de la información en las etiquetas/envases}

Como se observa en la Gráfica 4, el 58.3\% de los entrevistados se ubica en las categorías totalmente de acuerdo y de acuerdo respecto a que leen los ingredientes que contiene un producto; sin embargo hay una porción importante de los entrevistados que no han desarrollado este hábito, el cual les permitiría tomar decisiones mejor informadas.

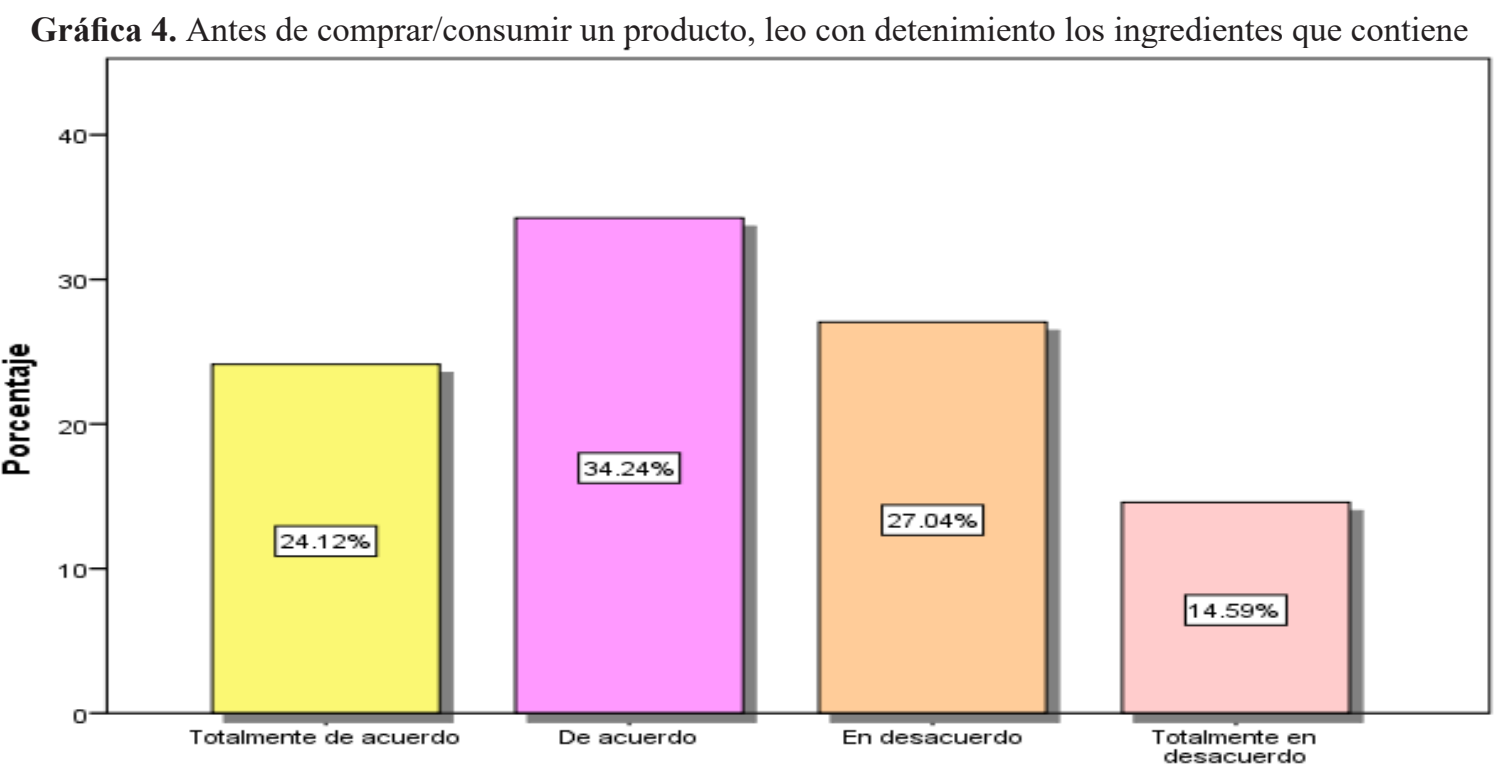

Fuente: elaboración propia

Respecto a la información nutricional que aparece en los sellos de advertencia, nuevamente la mayoría (56\%) de los entrevistados está de acuerdo en que ésta es clara; sin embargo también se observa un grupo importante de entrevistados que no la considera clara (Gráfica 5).

Gráfica 5. La información que aparece en los sellos negros de las etiquetas de los alimentos y bebidas proporcionan información nutricional clara

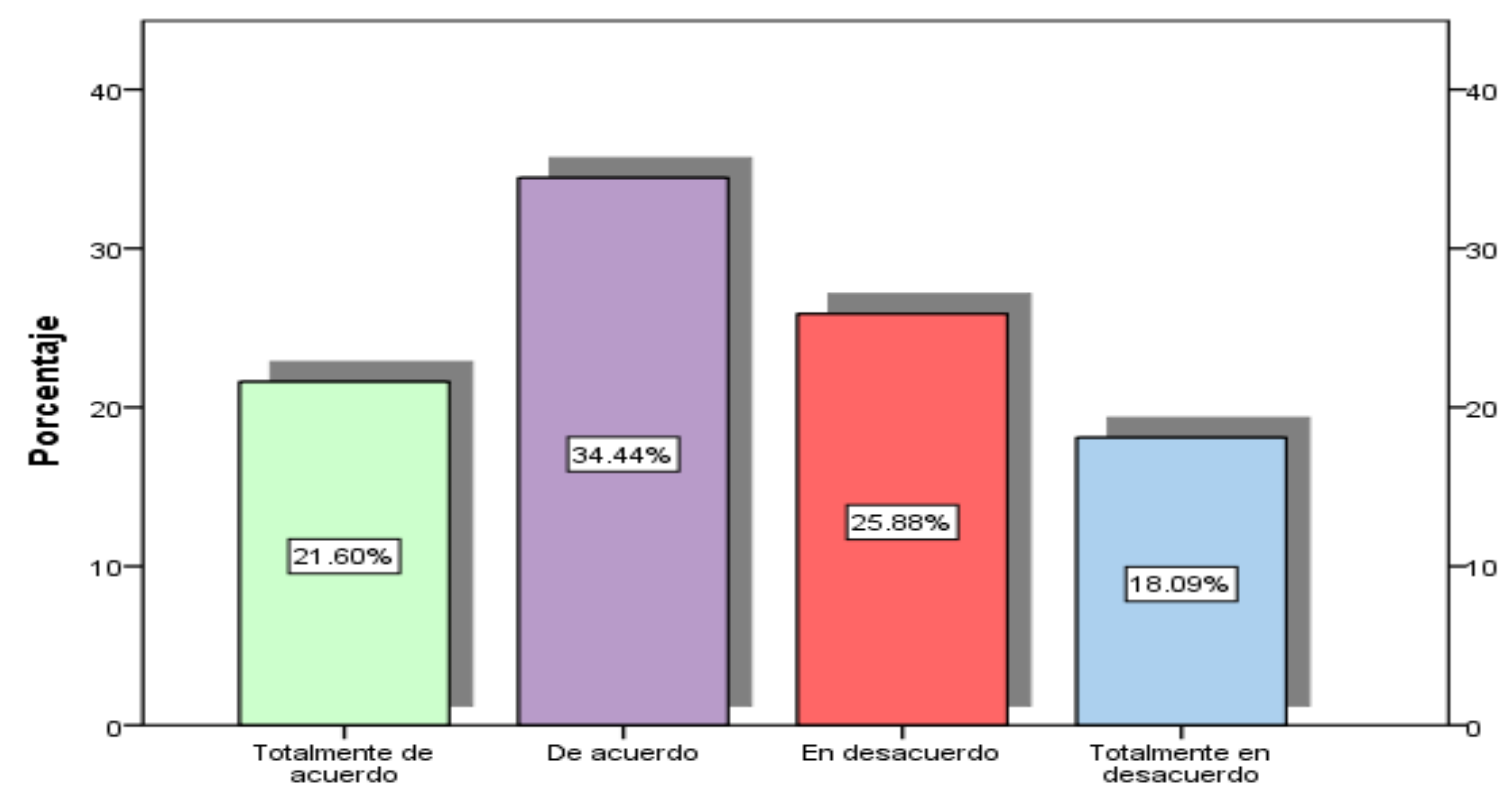

Fuente: elaboración propia 
Finalmente, respecto a este grupo de variables, el 70\% de los respondientes considera que los productos que se venden a granel, también deberían contar con los sellos de advertencia, ya que al no contenerlos, se podría generar una falsa impresión de que el contenido nutrimental es apropiado, y repercutir en decisiones de consumo no correctamente informadas.

\section{Influencia de los sellos en la compra y consumo de los productos}

Al preguntar a los entrevistados si todos los productos que consumen contienen sellos negros, el 55.8\% de las respuestas se ubica en las categorías totalmente en desacuerdo y en desacuerdo, lo cual podría implicar que una parte importante de la dieta de los consumidores entrevistados está conformada por productos no necesariamente procesados.

Como se aprecia en el Cuadro 4, la presencia de los sellos en los productos no motiva a los consumidores a dejar de comprarlos. Por otro lado, hay un efecto positivo de los sellos, en el sentido de que han hecho conscientes a los consumidores de que algunos productos que consideraban sanos, realmente no lo son (40.7\% totalmente de acuerdo). Sin embargo, en su opinión una buena alimentación no implica consumir únicamente productos que no contegan sellos $(63.2 \%$ en desacuerdo+totalmente en desacuerdo).

Cuadro 4. Influencia de los sellos al comprar/consumir productos

\begin{tabular}{|c|c|c|}
\hline Pregunta & Categoría 1 (\%) & Categoría $2(\%)$ \\
\hline $\begin{array}{l}\text { Dejo de comprar/consumir un producto } \\
\text { cuando leo la información de los sellos }\end{array}$ & $\begin{array}{l}\text { En desacuerdo } \\
\quad 34.2\end{array}$ & $\begin{array}{l}\text { Totalmente en } \\
\text { desacuerdo } \\
24.5\end{array}$ \\
\hline $\begin{array}{l}\text { Los sellos negros que aparecen en las } \\
\text { etiquetas/envases me desmotivan a } \\
\text { comprarlos/consumirlos }\end{array}$ & $\begin{array}{l}\text { En desacuerdo } \\
\quad 30.5\end{array}$ & $\begin{array}{l}\text { De acuerdo } \\
29.0\end{array}$ \\
\hline $\begin{array}{l}\text { Hay productos que consideraba sanos y con } \\
\text { los sellos me he dado cuenta que no lo son }\end{array}$ & $\begin{array}{l}\text { Totalmente de acuerdo } \\
\qquad 40.7\end{array}$ & $\begin{array}{l}\text { De acuerdo } \\
29.0\end{array}$ \\
\hline $\begin{array}{l}\text { Para tener una buena alimentación hay que } \\
\text { consumir solamente productos sin sellos }\end{array}$ & $\begin{array}{l}\text { En desacuerdo } \\
32.7\end{array}$ & $\begin{array}{l}\text { Totalmente en } \\
\text { desacuerdo } \\
30.5\end{array}$ \\
\hline
\end{tabular}

Fuente: elaboración propia

Una de las medidas introducidas para mejorar la alimentación infantil es la eliminación de personajes animados/superhéroes/deportistas en los empaques de los productos; sin embargo la mayoría de los entrevistados (62.5\%, categorías en desacuerdo y totalmente en desacuerdo) consideran que esta medida no implicará que los niños dejen de consumir los productos. 
Gráfica 6. Los niños al no ver personajes animados/superhéroes/deportistas en los empaques de los productos dejarán de

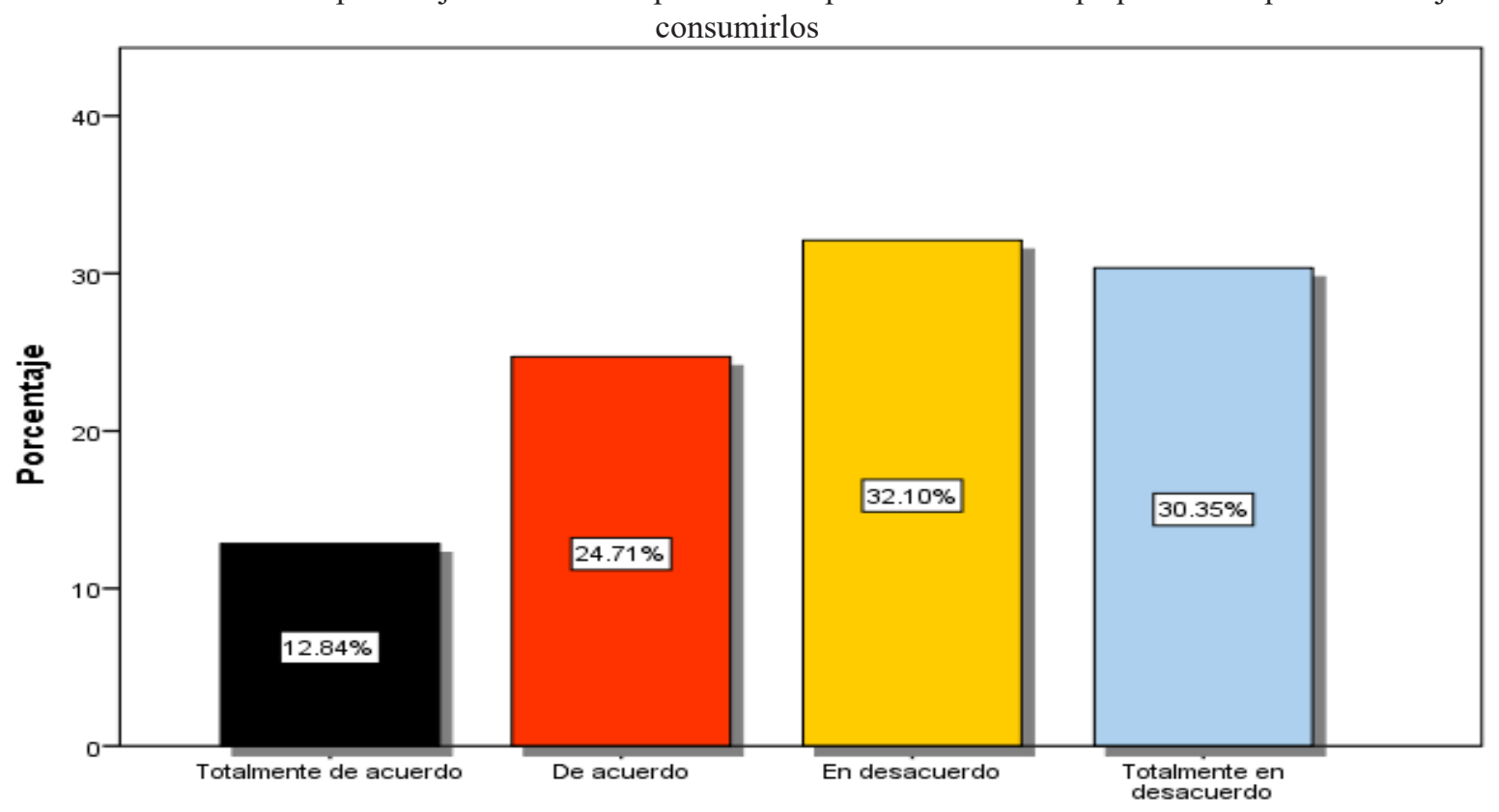

Fuente: elaboración propia

En opinión de la mayoría de los entrevistados los sellos de advertencia no son una buena medida para combatir la obesidad y el sobrepeso (63.2\%, categorías totalmente de acuerdo y de acuerdo) ni para mejorar la alimentación de los mexicanos (63.3\%, categorías totalmente de acuerdo y de acuerdo) como se muestra en el Cuadro 5. Asimismo, el 63.3\% (categorías totalmente de acuerdo y de acuerdo) considera que con el tiempo los consumidores se acostumbrarán a los sellos y no los tomarán en cuenta en sus decisiones de compra.

Cuadro 5. Efectos de las medidas para mejorar la alimentación de los consumidores

\begin{tabular}{|c|c|c|}
\hline Pregunta & Categoría 1 (\%) & Categoría 2 (\%) \\
\hline $\begin{array}{c}\text { Los sellos en los envases/empaques no son } \\
\text { una buena medida para combatir la obesidad } \\
\text { y el sobrepeso }\end{array}$ & Totalmente de acuerdo & De acuerdo \\
\hline $\begin{array}{c}\text { Los sellos negros en los alimentos/bebidas } \\
\text { no son una buena medida para mejorar la } \\
\text { alimentación }\end{array}$ & 32.7 & 30.5 \\
\hline $\begin{array}{c}\text { Con el tiempo los consumidores se } \\
\text { acostumbrarán a ver los sellos y no les } \\
\text { prestarán atención para decidir su compra }\end{array}$ & 36.8 & De acuerdo \\
& & 26.5 \\
\hline
\end{tabular}

Fuente: elaboración propia

\section{Prueba de Hipótesis}

Para llevar a cabo la prueba de hipótesis sobre la relación entre el conocimiento de los sellos de advertencia y la modificación de los hábitos de consumo de los alimentos en los que éstos aparecen, se contrastaron las siguientes variables, aplicando la prueba de $\mathrm{X}_{\mathrm{i}}^{2}$ : 
$\mathrm{H}_{01}$ : No existe relación entre el conocimiento de los sellos de advertencia y el que éstos desmotiven a los consumidores a comprar los productos.

$\mathrm{H}_{02:}$ No existe relación entre el conocimiento de los sellos de advertencia y el que los consumidores dejen de comprarlos al leer la información de los mismos.

En ambos casos a un nivel de significancia de .05 no se rechaza la hipótesis nula, por lo que se concluye que no hay relación entre el conocimiento de los sellos de advertencia y la modificación de los hábitos de consumo.

Con base en lo anterior puede decirse que los sellos han logrado captar la atención de los consumidores, pero no representan un estímulo lo suficientemente fuerte para dejar de comprar/consumir los productos en los que aparecen sellos de advertencia.

\section{DISCUSIÓN Y CONCLUSIONES}

Los hallazgos del presente estudio muestran que los sellos de advertencia han cumplido con el objetivo de llamar la atención de los consumidores hacia el contenido excesivo de ingredientes que resultan dañinos para la salud; sin embargo, no los motivan a cambiar sus hábitos. Adicionalmente, no los consideran una buena medida para combatir el sobrepeso y la obesidad, ni para mejorar la alimentación de los mexicanos; y señalan que con el tiempo la gente se acostumbrará a verlos, lo cual de alguna manera disminuirá su influencia en las decisiones de consumo.

Como se mencionó anteriormente, desde el sexenio del presidente Felipe Calderón se han estado planteando diversas iniciativas tendientes a revertir la tendencia del sobrepeso y la obesidad y sus consecuencias en la salud de los mexicanos, desafortunadamente, no se ha logrado este objetivo y el problema sigue creciendo.

En el caso de los sellos de advertencia es prematuro señalar el resultado que se alcanzará, aunque los hallazgos del presente estudio podrían ser un indicador de la necesidad de replantear la estrategia del gobierno mexicano. En este sentido, la solución de una problemática como la del sobrepeso y la obesidad demanda de un enfoque multidisciplinario en el campo del cambio social, y una disciplina que por su naturaleza puede contribuir es la mercadotecnia social, que ha sido utilizada con resultados positivos en otros países.

La mercadotecnia social plantea el desarrollo de programas integrales donde el objetivo central es un cambio de comportamiento, y se utilizan herramientas como la investigación de mercados, la identificación de los públicos objetivo, el planteamiento de intercambios lo suficientemente atractivos para modificar la conducta, así como la utilización de las cuatro P's. Si se analizan las acciones realizadas en México, entre ellas el etiquetado frontal de advertencia, se observa que para la mayoría de los consumidores el intercambio no es lo suficientemente atractivo para motivarlo al cambio; además de que en el caso de los sellos de advertencia se modifican las etiquetas/envases pero no van acompañados de una explicación clara para que el consumidor pueda interpretarlos correctamente y tomar decisiones informadas. En opinión de algunos nutriólogos el punto de partida debería ser educar a la población para que aprenda a comer bien.

Es pertinente aclarar, que un cambio de este nivel no se logra solo con campañas de mercadotecnia social, pero podría ser un primer paso para abordar esta problemática y crear consciencia entre la 
población de la importancia de un consumo responsable; y por otro lado, de la necesidad de prácticas socialmente responsables por parte de la industria alimentaria.

\section{REFERENCIAS}

1. Andreasen, A.R. (2002). Marketing social change in the social change marketing. Journal of Public Policy, 21 (1), pp. 3-13.

2. Andreasen, A.R. (2005). Social Marketing in the 21st Century. California. Sage: Thousand Oaks.

3. Alianza por la salud alimentaria (2019) Al sector salud la diabetes le cuesta 613 millones de dólares al año. https://alianzasalud.org.mx/

4. Barquera, S. y White, M. (2018). Treating Obesity Seriously in Mexico: Realizing, Much Too Late, Action Must Be Immediate. Obesity. 26 (10). pp. 1530-1531. https://doi/org/10.1002/oby.22296.

5. Dibb, S. and Carrigan, M. (2013). Social marketing transformed: Kotler, Polonsky and Hastings reflect on social marketing in a period of social change. European Journal of Marketing, 47 (9), pp. 1376-1398.

6. DOF. (2020). Modificación a la Norma Oficial Mexicana NOM.051, Especificaciones generales de etiquetado para alimentos y bebidas no alcohólicas preenvasados-Información comercial y sanitaria. México.

7. Domegan, C.T. (2008). Social marketing: implications for contemporary marketing practices classification scheme. Journal of Business \& Industrial Marketing. 23 (2), pp. 135-141.

8. Encuesta Nacional de Salud y Nutrición (2018). ensanut.insp.mx>ensanut_2018_presentacion_ resultados

9. Instituto Nacional de Salud Pública (2020). Sobrepeso y obesidad en México www.insp.mx > 4737-sobrepeso-obesidad-mexic

10. Lusch, R.F. y Vargo, S.L. (2006). Service-dominant logic: reactions, reflections and refinements. Marketing Theory. 6 (3), pp. 281-288.

11. Poder del consumidor (2020). La obesidad en México es alarmante, urge fortalecer las políticas: OCDE www.poderdelconsumidor.org/2020/01/la-obesidad-en-mexico-es-al

12. Stead, M., Hastings, G. y McDermott, L. (2007). The meaning, effectiveness and future of social marketing. Obesity reviews 8 (Suppl.1) 189-193.

13. Villanueva, D. (2020). México, el país con mayor obesidad de AL: OCDE https://www.jornada. com.mx/ultimas/economia/ 